\title{
RIESGOS PSICOSOCIALES DERIVADOS DE LA PRECARIEDAD LABORAL EN LA CAV: POSIBLES LÍNEAS DE ACTUACIÓN
}

\author{
Aida López Cabrera
}

DOI: $10.1387 /$ lan-harremanak.15427

\section{ABSTRACT}

Los cambios socioeconómicos de los últimos años han dado lugar a una reconfiguración del mundo laboral tendente a flexibilizar el mercado de trabajo. Estos cambios no solo disminuyen la calidad y cantidad de empleo, sino que también merman la salud mental de los trabajadores y aumenta la probabilidad de exposición a riesgos psicosociales. Para entender este cambio y contextualizar la precariedad latente en la actualidad, se abordarán las distintas transformaciones de las relaciones laborales desde el siglo XVI hasta nuestros días.

Se analizará el término de precariedad y se estudiaran las distintas dimensiones de este complejo fenómeno: flexibilidad externa, flexibilidad interna, calidad y seguridad. Junto a esto, se explicará de manera breve los cambios normativos introducidos por el ejecutivo en aras a fomentar los empleos precarios.

De la misma forma, se contextualizarán y se definirán los riesgos psicosociales, como también los factores de los mismos relacionados con la precariedad, prestando especial atención al estrés como riesgo psicosocial predominante en el caso que nos ocupa. Se analizará la regulación existente en materia de riesgos psicosociales a nivel europeo y estatal con la finalidad de identificar sus posibles deficiencias.

La falta de regulación a cerca de los riesgos psicosociales, la poca concienciación social y el modelo productivo que promueve la maximización de beneficios a cualquier pre- 
cio, generan una despreocupación por los trabajadores y su salud. Se vislumbra pues, la necesidad de crear una nueva cultura socioeconómica y una serie de ajustes legislativos para poder combatir la generación emergente de riesgos de carácter psicosocial en el ámbito laboral.

Palabras clave: Precariedad, riesgos psicosociales, estrés, cambios normativos, economía social y solidaria.

The socio-economic changes in recent years have led to a reconfiguration of the workplace aimed at the labour market making it more flexible. These changes not only reduce the quality and quantity of employment, but also undermine the mental health of workers and increase the likelihood of exposure to psychosocial risks. To understand this change and contextualize the latent precariousness at present, the various transformations of labour relations will be addressed from the sixteenth century to the present days.

The term precariousness will be analysed and the different aspects of this complex phenomenon will be studied. Beside, normative changes introduced by the executive will be briefly explained and current data will be examined.

In the same way, the psychosocial risks and their factors will be defined and contextualized, specially taking into account the stress. The European and Spanish psychosocial risks' regulation will be analysed in order to identify possible failures.

These two concepts' interrelation will be addressed from experiential point of view through qualitative study principally focused on the situation of qualified young workers.

The regulation lack about psychosocial risks, the little social awareness and the capitalist productive model produce carelessness on workers and their health. Therefore, it is necessary to create a new socioeconomical culture and legislative adjustments in order to fight emerging generation of psychosocial risks in the labour field.

Key Words: Precariousness, psychosocial risk, stress, regulatory changes, social and solidary economy. 


\section{La precariedad en el empleo}

\subsection{Marco histórico}

A lo largo de la historia las relaciones de trabajo se han configurado en base a un modo de producción determinado y al modelo socioeconómico resultante. Es decir, los vínculos establecidos entre trabajo y capital dentro de un proceso productivo dependen de una estructura social concreta y de las relaciones de poder existentes en ella, como también del sistema económico predominante.

Las relaciones laborales propiamente dichas se originan en el siglo xv y xvI junto con una serie de importantes transformaciones sociales, culturales, económicas y políticas. Nace el mercantilismo, donde encontramos por primera vez la figura del trabajador asalariado definido como una persona libre que vende su fuerza de trabajo a cambio de un salario. La propiedad del producto terminado es posesión del patrón capitalista, el cual se sirve de su venta para generar un capital para la acumulación.

A mediados del siglo xviII se inicia en Gran Bretaña la denominada revolución industrial, que se extendió al resto de Europa y, que trajo como consecuencia la instauración del modo de producción capitalista dentro del marco del liberalismo económico promulgado por Adam Smith. De este modo, el trabajo manual pasa a ser mecánico, el núcleo del proceso productivo pasa a ser la máquina, y el trabajador debe únicamente amoldarse a su ritmo de actividad y a sus exigencias. Se cristaliza una fuerte división técnica del trabajo y una intensificación del mismo para hacer frente a las exigencias de un mercado altamente competitivo y desregularizado.

Para dar respuesta a las carencias del modelo liberal, aparece tras la segunda guerra mundial el keynesianismo, que defiende la actuación sobre la demanda mediante el incremento de consumo público y privado con el fin de crear pleno empleo y un mercado de masas. Junto con esta corriente, nace la relación salarial fordista, caracterizada por contratos de trabajo estables y remuneraciones según tiempo de trabajo y precios de consumo. De esta manera se crea un consumo de masas, se da una fusión de la figura del trabajador y el cliente. En contrapartida de esta relación salarial, las organizaciones sindicales que agrupan al perso- 
nal asalariado, negocian colectivamente con la dirección de las empresas, lo que provoca una etapa de gran cohesión y concordia social.

Sin embargo, esta doctrina muestra sus limitaciones ante los cambios generados por la crisis de los 70. Esto da lugar a una replantación y reformulación de las políticas liberales clásicas (neoliberalismo) incrementándose la incertidumbre y la mutabilidad de los mercados de mano de una diversificación y globalización de la demanda. Esto, junto a la introducción de nuevas tecnologías, provoca una reorganización de las empresas tendentes a flexibilizar las relaciones de trabajo. Esta flexibilización supone un nuevo modelo de organización que pretende asegurar la producción con menos asalariados efectivos, y da lugar a un empleo segmentado e inestable. Junto a esto, surge la desestabilización de los sistemas de protección social, que se convierte en un factor más de amenaza a la seguridad y al control del futuro de la sociedad. El Estado deja de ser garantía de estabilidad, la cual es gestionada de manera personal y privada.

\subsection{Concepto y formas. una aproximación a la multidimesionalidad del empleo precario}

El empleo precario es un concepto significativamente complejo puesto que no existe una definición uniforme a nivel internacional que nos permita realizar una evaluación escueta de esta problemática. Más aun, existen distintas concepciones sobre la precariedad en los distintos mercados de trabajo nacionales y dependiendo de las perspectivas teóricas desde donde se abordan. La vulnerabilidad que desprende el concepto deviene de una norma social (las relaciones laborales aceptadas como estándar) y de un momento histórico determinado, y demuestra que existe la necesidad de identificar las principales dimensiones de este fenómeno para asentar el pilar para una definición universal.

Trabajo precario se refiere a

Empleo caracterizado por contratos de trabajo atípicos, con limitados o inexistentes beneficios sociales y nulas prestaciones, un elevado nivel de inseguridad laboral, reducido nivel de permanencia en el empleo, bajos salarios y un alto riesgo de lesiones y enfermedades profesionales. Desde el punto de vista del trabajador, el trabajo precario se refiere a trabajo incierto, impredecible y riesgoso (Evans y Gibb, 2009).

A finales del siglo $\mathrm{xx}$ se formula el concepto de trabajo decente, reconociéndose el trabajo como fuente de dignidad personal, estabilidad familiar, paz en la comunidad, democracias que actúan en beneficio de todos, y crecimiento económico, que aumenta las oportunidades de trabajo productivo y el desarrollo de las empresas. Al mismo tiempo se reconoce que el trabajo decente consta de cinco elementos: Empleos productivos y protegidos, con retribuciones jus- 
tas, regidos por normas laborales sustentadas en los derechos fundamentales en el trabajo, sin discriminaciones de ningún tipo y en contextos donde el diálogo social y la negociación colectiva sean mecanismos plenamente vigentes (Somavía, 1999).

En Espańa existe la tendencia a ligar el término precariedad con el de flexibilidad, sin embargo, a continuación, trataré de establecer de manera resumida un análisis multidimensional de la precariedad para clarificar el término, superando esta limitación conceptual mediante una perspectiva tridimensional de flexibilidad/calidad/seguridad:

Tabla 1

\begin{tabular}{c|cc}
\hline Dimensiones & Clases & Manifestaciones \\
\hline $\begin{array}{c}\text { Flexibilidad } \\
\text { externa }\end{array}$ & $\begin{array}{c}\text { Desempleo } \\
\text { Contratación temporal } \\
\text { Subcontratación }\end{array}$ & $\begin{array}{c}\text { Contratos formación, fin de obra... } \\
\text { ETTs, falsos autónomos... }\end{array}$ \\
\hline $\begin{array}{c}\text { Flexibilidad } \\
\text { interna }\end{array}$ & $\begin{array}{c}\text { Tiempo de trabajo } \\
\text { Funciones } \\
\text { Salario }\end{array}$ & $\begin{array}{c}\text { Media jornada, horarios variables, teletrabajo... } \\
\text { Movilidad geográfica y funcional } \\
\text { Salario a comisión, estancamiento... }\end{array}$ \\
\hline Calidad & $\begin{array}{c}\text { Formación } \\
\text { Promoción y ascenso }\end{array}$ & $\begin{array}{c}\text { Nula posibilidad de desarrollo de carrera } \\
\text { Carga laboral excesiva/insuficiente }\end{array}$ \\
\hline Seguridad & Protección Social & Falta de protección frente al despido, accidentes, \\
desempleo...
\end{tabular}

Fuente: Elaboración propia.

\subsection{Medidas flexibilizadoras introducidas en la legislación laboral española en los ultimos ańos}

Con la finalidad de superar la crisis de un sistema desgastado, se han realizado grandes reformas legislativas en materia sociolaboral y económica. En líneas generales, todos estos cambios han dado lugar a una flexibilización notable en el sistema de relaciones laborales y a una tendencia a favorecer a los empresarios y debilitar enormemente el poder de la negociación colectiva. A continuación se presentan de manera resumida los cambios normativos más relevantes realizados en los últimos años para favorecer la instauración de empleos precarios: 


\subsubsection{Flexibilidad externa}

Se introducen dos nuevas modalidades contractuales: el contrato de trabajo a tiempo parcial con vinculación formativa ${ }^{1} \mathrm{y}$ el contrato primer empleo joven ${ }^{2}$. Ambos contratos vienen acompañados de un amplio conjunto de bonificaciones para las empresas que decidan realizarlos.

En cuanto, a las alteraciones en el contrato para la formación y el aprendizaje, la edad para celebrar el contrato se extiende hasta los treinta hasta que la tasa de desempleo se sitúe por debajo del $15 \%^{3}$, se extiende hasta los tres años la duración máxima estipulada, se da luz verde a la prorroga en los casos en los que los contratos se hayan celebrado por un tiempo inferior al máximo establecido, se permite la realización de un segundo contrato con la misma empresa en los casos en los que el fin sea la obtención de una cualificación distinta a la del objeto del anterior, y se amplía el tiempo de trabajo efectivo compatible con la formación.

Se suprime el art. 15.5 ET el cual establecía que se considerarían trabajadores fijos a aquellos que dentro de treinta meses hubieran estado veinticuatro de ellos contratados mediante dos o más contratos temporales. Se vislumbra así, una clara aceptación de encadenamientos de contratos temporales ${ }^{4}$.

Se amplía el alcance de los contratos de puesta a disposición, permitiendo que las ETTs puedan recurrir a contratos de primer empleo joven ${ }^{5}$, de formación y aprendizaje y de prácticas ${ }^{6}$. También se les permite actuar como agencias de colocación 7 .

\subsubsection{Flexibilidad interna}

En materia de tiempo de trabajo son dignas de mención las siguientes modificaciones:

—El número máximo de horas permitidas en términos absolutos en los contratos a tiempo parcial y de relevo pasa a ser del $30 \%$ de las horas ordinarias, pudiendo alcanzar el $60 \%$ por negociación colectiva, sin que la suma de estas y las horas ordinarias puedan superar la jornada completa,

1 Art. 9 Ley 11/2013, de 26 de julio, de medidas de apoyo al emprendedor y de estímulo del crecimiento y de la creación de empleo.

2 Art. 12 Ley 11/2013, de 26 de julio, de medidas de apoyo al emprendedor y de estímulo del crecimiento y de la creación de empleo.

${ }^{3}$ Disposición transitoria novena. Ley 3/2012, de 6 de julio, de medidas urgentes para la reforma del mercado laboral.

${ }^{4}$ Art. 17 Ley 3/2012.

5 Disposición adicional quinta. Ley 11/2013.

${ }^{6}$ Art. 3 Real Decreto Ley 16/2013, de 20 de diciembre, de medidas para favorecer la contratación estable y mejorar la empleabilidad de los trabajadores.

7 Art. 1 Ley $3 / 2012$. 
o en su defecto, la jornada máxima legal, suprimiéndose el límite del $77 \%$ que fijaba el ET anteriormente . $^{8}$

- Ampliación de un 5\% a un $10 \%$ la jornada que puede distribuirse anualmente de manera irregular en defecto de convenio colectivo o acuerdo de empresa, y siempre que se preavise la trabajador con una antelación de cinco días?.

En relación con el salario percibido por el trabajador debemos hacer referencia al art. 4 de la Carta Social Europea. Esta disposición reconoce el derecho de todo trabajador a una remuneración suficiente para llevar un nivel de vida digo, reconociéndose está como la equivalente al $50 \%$ del nivel medio de los salarios del Estado (Comité Europeo de Derechos Sociales, 2008). El órgano encargado del cumplimiento de estas obligaciones, El Comité Europeo de Derechos Sociales, ha considerado que el SMI establecido por la Ley de Presupuestos Generales del Estado ${ }^{10}$ no asegura una vida decente a los trabajadores, teniendo en consideración las contribuciones a la Seguridad Social y la deducción de la aportación al impuesto sobre la renta. Asimismo, ha demandado información sobre la remuneración mínima de los autónomos dependientes y la cobertura de los convenios colectivos en esta materia.

Por lo que se refiere a la movilidad funcional y geográfica, se han dado grandes cambios en estos últimos años con el fin de ceder al empresario mayor flexibilidad.

En cuanto al cambio de las bases del sistema de clasificación profesional, se suprimen las categorías profesionales, limitándose únicamente a un sistema basado en grupos profesionales:

—Equiparación de las funciones a un determinado grupo guiándose por el concepto de "funciones prevalentes».

- Modificación del concepto de grupo profesional introduciendo, además de distintas funciones y especialidades profesionales, «distintas tareas $o$ responsabilidades asignadas al trabajadon ${ }^{11}$. Se da un plazo de año para que los convenios colectivos vigentes adapten sus sistemas de clasificación profesional a lo establecido por la nueva redacción ${ }^{12}$.

- Se elimina la posibilidad de control de la autoridad laboral que le concedía el at. 40 ET. Se amplían los casos de movilidad justificada de manera ilimitada. Esta justificación también afecta a las reducciones y a las suspensiones del tiempo de trabajo $^{13}$.

8 Art. 1 R.D.-ley 16/2013, de 20 de diciembre, de medidas para favorecer la contratación estable y mejorar la empleabilidad de los trabajadores.

9 Art. 9 Ley $3 / 2012$.

10 Ley 36/2014, de 26 de diciembre, de Presupuestos Generales del Estado para el año 2015.

11 Art. 8 Ley $3 / 2012$.

12 Disposición adicional 9. . ${ }^{\mathrm{a}}$ Ley $3 / 2012$.

13 Art. 13 Ley 3/2012. 
- Cambios en los criterios de preferencia de permanencia en la empresa, se pasa a evitar el traslado de los colectivos con situación más riesgosa en caso de darse esa movilidad ${ }^{14}$.

\subsubsection{Seguridad}

Se facilita el despido realizando los siguientes cambios normativos:

- Se suprime la necesidad de la autorización previa de la autoridad laboral establecida en el art. 51 ET para el caso de los despidos colectivos, aunque si plantea la obligatoriedad de un periodo de consultas entre representantes de la empresa y de los trabajadores.

- Posibilidad de despido objetivo del trabajador por faltas de absentismo que supongan el $20 \%$ de las jornadas hábiles en dos meses, aunque estas sean justificadas. En casos de no adaptación al trabajo se elimina el plazo de adaptación base (antes de dos meses) y se eliminan los límites del periodo de esa formación (antes de tres meses).

- Disminución de la indemnización por despido improcedente. Asimismo, si el empresario opta por la readmisión el trabajador no tendrá derecho a los salarios de tramitación ${ }^{15}$.

- Se permite el periodo de prueba de un año ${ }^{16}$ para el nuevo contrato indefinido de apoyo a emprendedores que crea el legislador hasta que la tasa de desempleo se situé por debajo del $15 \%{ }^{17}$.

- Se establece la interrupción de este periodo para los casos de incapacidad temporal, riesgo durante el embarazo, maternidad, y adopción o acogimiento, riesgo durante la lactancia y paternidad ${ }^{18}$.

Mediante el art. 14 de la Ley 3 /2012 se introducen las siguientes transformaciones en el régimen de la negociación colectiva:

— Eliminación de plazos máximos para la denuncia y para la negociación de la renovación de convenios colectivos.

- Establecimiento de un nuevo procedimiento para la modificación sustancial de condiciones de trabajo.

- Se permite la inaplicación de convenios colectivos, tanto de sector como de empresa, cuando concurran causas económicas, técnicas, organizativas o productivas.

\footnotetext{
14 Art. 11 Ley 3/2012.

15 Art. 8 Ley 3/2012.

16 Art 4.3 Ley 3/2012.

17 Art. 2 RDL 16/2013.

18 Art. 1.2 del RDL 16/2013.
} 
- Se establece la prioridad de los acuerdos de empresa frente a los acuerdos sectoriales en las siguientes materias: Salario, tiempo de trabajo, clasificación profesional, modalidad contractual y medidas de conciliación.

— Establecimiento de un plazo máximo de dos años de ultraactividad del convenio, aplicándose en su lugar el convenio de ámbito superior si lo hubiera.

- Aligeramiento del contenido mínimo de los convenios colectivos, cambiando el papel de las comisiones paritarias, cuyas funciones quedan condicionadas a lo que se acuerde en la negociación colectiva.

Podemos apreciar una profunda alteración de la negociación colectiva, que ha sido uno de los más afectados por los cambios normativos recientes.

Se apuesta decididamente por la descentralización de la negociación colectiva, primando a las actuaciones del ámbito empresarial con el fin de promocionar su adaptabilidad a las exigencias del mercado en materias clave para la competitividad y la productividad empresarial. Vemos, de alguna manera, una tendencia monopolística, puesto que a la empresa se le atribuye mayor poder contractual para fijar las condiciones de trabajo y los instrumentos necesarios para poder desentenderse de la negociación sectorial. Esto genera, principalmente en pequeñas y medianas unidades productivas, la desprotección jurídica del trabajador dada la escasa implantación de los sindicatos. De igual modo, los nuevos ajustes en la ultraactividad de los convenios colectivos fomentan la regulación a la baja de las condiciones de trabajo al exponer a los trabajadores a convenios marco o al Estatuto de los Trabajadores.

El Comité Europeo de Derechos Sociales también se ha pronunciado sobre las medidas adoptadas en la negociación colectiva, demandando la supresión de derechos de los trabajadores, y reconociendo únicamente la legitimidad de los convenios de empresa en los casos en los que la decisión sea asumida por los propios sindicatos.

\section{Riesgos psicosociales derivados de la precariedad en el empleo}

\subsection{La precariedad como factor de riesgo psicosocial y el estrés laboral}

Tradicionalmente, se ha considerado los factores físicos, químicos y biológicos como el foco de la amenaza para la salud de los trabajadores. Se ha venido reconociendo que estos factores son los causantes de la mayor parte de accidentes y enfermedades vinculadas al trabajo. Sin embargo, en la última década estamos asistiendo a un cambio de paradigma en el ámbito laboral que hace que surjan nuevos desafíos para la seguridad y la salud de los trabajadores.

Todos estos cambios suponen transformaciones importantes en la actividad laboral, en la forma de organizarla y en las propias empresas en las que esta se 
lleva a cabo. En este contexto, es imprescindible recordar que el empleo plantea demandas emocionales, intelectuales y sociales que deben ser igualmente protegidas y estudiadas para garantizar la seguridad y salud de los trabajadores.

Uno de los primeros documentos oficiales e internacionales en abordar el tema de los factores psicosociales comienza por reconocer la complejidad del tema y propone la siguiente definición:

Los factores psicosociales en el trabajo consisten en interacciones entre el trabajo, su medio ambiente, la satisfacción en el trabajo y las condiciones de organización, por una parte, y por la otra, las capacidades del trabajador, sus necesidades, su cultura y su situación personal fuera del trabajo, todo lo cual, a través de percepciones y experiencias, puede influir en la salud, en el rendimiento y en la satisfacción en el trabajo (OIT, 1986: 3).

Estos factores generan la posibilidad de que un trabajador sufra un determinado daño derivado del trabajo, es decir, crean un riesgo para la integridad y salud mental y psicológica de los trabajadores en una organización. A estos posibles daños les denominamos riesgos psicosociales.

Partiendo de la idea aportada por Lazarus el estrés no solo depende del conjunto de factores que rodean el mundo del trabajo, sino que también juega un papel esencial la percepción de los individuos de esos factores y de la habilidad que poseen para adaptarse a los mismos. Como podemos ver en la siguiente ilus-

Figura 1
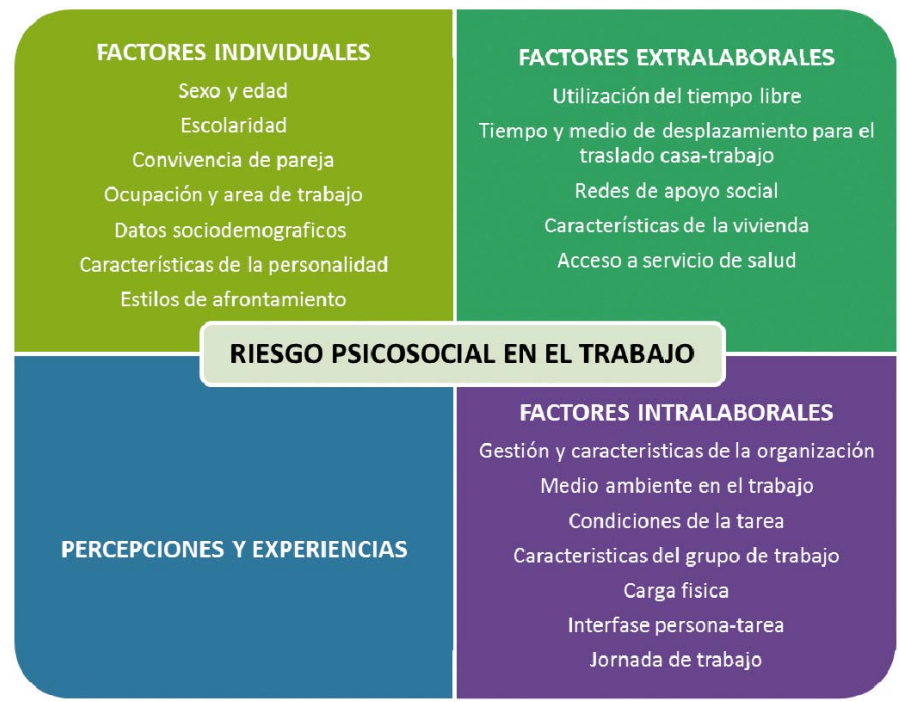

Fuente: Elaboración propia. 
tración, los riesgos psicosociales se gestan por la interrelación de factores tanto laborales como extralaborales e individuales.

Cuando la demanda del ambiente (ya sea laboral o extralaboral) es excesiva frente a los recursos de afrontamiento que posee el trabajador, se desarrolla una situación de estrés en la que el individuo intentará generar más recursos para atender las demandas de la situación.

Autores como McGrath (1970) define el estrés como un desequilibrio sustancial percibido entre la demanda y la capacidad de respuesta del individuo bajo condiciones en las que le fracaso ante esta demanda posee importantes consecuencias percibidas.

Ante los agentes estresores las personas activan una respuesta fisiológica, psicológica y de comportamiento susceptibles de causar enfermedades tanto físicas como psicológicas; o en su caso, de potenciar enfermedades ya existentes por su carácter multifactorial.

Según expertos de la Asociación Española de Especialistas en Medicina del Trabajo (AEEMT) una de cada cuatro bajas laborales están asociadas al estrés laboral, y un $62 \%$ de los trabajadores han aumentado su nivel de estrés en el último año debido a la crisis, una mayor carga de trabajo, ajustes de plantilla o a la incertidumbre laboral ${ }^{19}$.

Centrándonos en las distintas dimensiones de la precariedad, vamos a analizar la interrelación entre la precariedad y los elementos generadores de riesgo psicosocial en el trabajo.

Tabla 2

\begin{tabular}{c|c}
\hline $\begin{array}{c}\text { Características } \\
\text { del trabajo }\end{array}$ & $\begin{array}{c}\text { Horarios atípicos e intempestivos } \\
\text { Falta de participación y control } \\
\text { Imposibilidad de conciliación }\end{array}$ \\
\hline Contexto laboral & $\begin{array}{c}\text { Baja remuneración o salario a destajo } \\
\text { Falta de perspectivas profesionales } \\
\text { Inseguridad laboral }\end{array}$ \\
Papel y cultura & Excusa o falta de capacidad para el puesto \\
\hline de la organización & Falta de identificación del responsable \\
Comunicación nula
\end{tabular}

Fuente: Elaboración propia.

19 http://www.elmundo.es/elmundosalud/2012/09/19/neurociencia/1348056989.html 


\subsection{Análisis jurídico}

Los riesgos psicosociales han sufrido una evolución en su consideración, un reconocimiento progresivo. A pesar de ello, la intervención normativa en esta materia es aún insuficiente, aunque va adquiriendo cada vez más relevancia social.

\subsubsection{Unión europea}

Dentro del conjunto de tratados que forman el contenido fundamental del conjunto de la Unión Europea, encontramos relevante mencionar para este caso el Tratado Constitutivo de la Comunidad Europea ${ }^{20}$ y la Carta de los Derechos Fundamentales ${ }^{21}$.

En el tratado anteriormente mencionado, se desarrollan las políticas y acciones comunitarias y los principios que rigen las mismas. Dos de estos principios (art. 2 y 3.1 y p)) recogen el deber de la comunidad de promover un alto nivel de protección y de mejora de la calidad del medio ambiente; la elevación del nivel y de la calidad de vida, y de la cohesión económica y social; como de establecer una política en el ámbito del medio ambiente y contribuir al logro de un alto nivel de protección de la salud.

Lo que la Comunidad pretende con sus acciones (art. 152.1) es garantizar un alto nivel de protección de la salud humana, previniendo enfermedades, evitando las fuentes de peligro, y apoyando la investigación, la información y la educación sanitarias. Asimismo, el art. 95.3 del mismo tratado nos dice que se tendrán en cuenta cualquier novedad basada en hechos científicos a la hora de actuar. Cabe destacar los fundamentos 11 y 12 de la sentencia de 15 de noviembre de 2001 del tribunal de justicia de la UE (ASUNTO C-49/00) que nos dice que

Es preciso, asimismo, indicar que los riesgos profesionales que han de ser objeto de una evaluación por parte de los empresarios no están determinados definitivamente, sino que evolucionan de forma constante en función, especialmente, del desarrollo progresivo de las condiciones de trabajo y de las investigaciones científicas en materia de riesgos profesionales.

Podemos deducir que el empresario no debe acotar su actividad preventiva únicamente a los riesgos físicos, biológicos y químicos tradicionales, sino que también debe incluir acciones tendentes a proteger a los trabajadores de los riesgos psicosociales y ergonómicos emergentes en la sociedad contemporánea.

20 Comunidad Europea (2002). Versión consolidada del tratado constitutivo de la Comunidad Europea, Niza, Diario Oficial de las Comunidades Europeas.

21 Comunidad Europea (2000). Carta de los derechos fundamentales de la Unión Europea, Niza, Diario Oficial de las Comunidades Europeas. 
Los arts. 136 y 137, en semejanza con el derecho fundamental del derecho al trabajo respetuoso con la salud seguridad y dignidad reconocido en el art. 31 de la Carta de derechos Fundamentales de la UE establece, entre otros, los siguientes objetivos comunitarios: fomento del empleo, mejora de las condiciones de vida y de trabajo, una protección social adecuada, diálogo social, y desarrollo sostenible y estable de los recursos humanos. Para su consecución, la UE se compromete a apoyar y complementar las actuaciones de los estados mediante iniciativas y evaluaciones, como de directivas que establezcan disposiciones mínimas que habrán de aplicarse de manera progresiva dependiendo de las circunstancias y reglamentaciones técnicas de los Estados miembros.

En este sentido, el 12 de junio de 1989 se aprobó la Directiva Marco 89/391/CEE del Consejo, relativa a la aplicación de medidas para promover la mejora de la seguridad y de la salud de los trabajadores en el trabajo. Esta disposición obliga al empresario a proteger a sus trabajadores frente a los riesgos derivados del trabajo, garantizando la seguridad y la salud de los trabajadores en todos los aspectos relacionados con el trabajo, y en colaboración con los mismos trabajadores y/o sus representantes. De la misma manera, se establecen criterios o principios que deben regir la actividad preventiva (eliminación o reducción de los riesgos en su origen, protección colectiva frente a la individual, etc.); la exigencia de actividades de control (vigilancia de la salud de los trabajadores, actuación frente a las emergencias, investigación de accidentes, información y formación de los trabajadores, etc.); y la planificación de una prevención integral y conjunta teniendo en cuanta el tipo y magnitud de los riesgos existentes en la empresa.

\subsubsection{España}

La Constitución Española (CE) nos ofrece el punto de partida de los derechos de todos los ciudadanos, como también de la determinación de competencias entre la Administración General del Estado y las Comunidades Autónomas.

El precepto base de nuestro país reconoce a todos los ciudadanos el derecho fundamental a la vida y a la integridad física y psíquica (art 15), como también el de respeto a la dignidad (art. 10). De igual forma, se reconoce el derecho a la salud (art. 43), y al disfrute de un medio ambiente adecuado para el desarrollo de la persona (art. 45.1). Para proteger estos derechos, los organismos públicos deben velar por la salud e higiene en el trabajo (40.2). De este último artículo se concluye que la responsabilidad de este ámbito recae en la Administración General.

Para el caso concreto de nuestra Comunidad Autónoma, el art. 12.2. del Estatuto de Autonomía ${ }^{22}$ concede competencias de ejecución de la legislación la-

22 Ley Orgánica 3/1979, de 18 de diciembre, de Estatuto de Autonomía para el País Vasco. 
boral del Estado, entendiéndose que los organismos públicos vascos pueden llevar a cabo medidas de prevención, asesoramiento técnico, vigilancia y control de la legislación en materia de prevención de riesgos y régimen sancionador, peor no tienen capacidad de reglamentación.

No podemos olvidar la Ley sobre Infracciones y Sanciones del Orden Social $^{23}$ donde se tipifican en los arts. 11, 12 y 13 las infracciones por acción u omisión que el empresario pudiese cometer en materia de prevención de riesgos laborales.

\subsubsection{Legislación específica: Ley de Prevención de Riesgos Laborales y Reglamento de los Servicios de Prevención}

Podemos decir que tanto la LPRL como el RSP intentan ofrecer una visión genérica e integradora del deber de protección de los trabajadores, superando una mirada tradicional que tiende a tener en cuenta únicamente los riesgos físicos, biológicos y químicos. El empresario debe garantizas la salud de sus trabajadores en todos los aspectos relacionados con el trabajo (14.2 LPRL) debiéndose integrar la actividad preventiva

Tanto en los procesos técnicos, en la organización del trabajo y en las condiciones en que éste se preste, como en la línea jerárquica de la empresa, incluidos todos los niveles de la misma (1.1 RSP).

Se obliga a que esa protección se realice mediante la utilización de la evaluación de riesgos y la planificación de medidas (art 16.1 LPRL y art. 1.1. RSP), y a valorar los riesgos identificados utilizando criterios objetivos que proporcionen un resultado veraz. Para entenderse como tal el resultado debe sobrevenir de un procedimiento o método establecido en una normativa específica si lo hubiera, o en su caso, de normas UNE o internacionales, o de guías de entidades u organizaciones de reconocido prestigio (5 RSP). En este sentido, la evaluación de los aspectos psicosociales se enfrenta a dos obstáculos: la dificultad de identificas el factor del que provienen o por el que pueden ser causados y la inexistencia de métodos legalmente previstos para su correcta y completa evaluación.

Para garantizar la protección de los riesgos identificados la ley prevé que los servicios de prevención cuenten con al menos un técnico superior especialista en ergonomía y psicosociología aplicada (15.2 y 15.4 RSP).

En cuanto al seguimiento de la correcta actuación preventiva y protectora de las empresas el arts. 29 y 30.4 RSP exige que las mismas sean auditadas cada cuatro años, y cada dos en los casos en los que la actividad sea especialmente peligrosa.

23 Real Decreto Legislativo 5/2000, de 4 de agosto, por el que se aprueba el Texto Refundido de la Ley sobre Infracciones y Sanciones en el Orden Social. 


\subsubsection{Estatuto de los Trabajadores}

El ET reconoce en su art. 4.2.d) y 19.1 el derecho de los trabajadores a gozar de una adecuada y eficiente política de seguridad e higiene y a su integridad física. Para contribuir a dicha protección el empresario está obligado a formarlos adecuadamente en materia de seguridad e higiene, especialmente en los casos en los que se realicen cambios de puestos y/o se apliquen nuevas técnicas susceptibles de causar nuevos riesgos (art. 19.4). De la misma forma, se protege la dignidad del trabajador al establecerse los límites de actuación del empresario a la hora de tomar medidas de vigilancia y control (art 20.3).

El incumplimiento por parte del empresario de estos deberes podrá ser razón justificada para que el trabajador pueda solicitar la extinción de la relación contractual (art. 50.1.c)). En tal caso, el trabajador tendrá derecho a ser indemnizado como si de un despido improcedente se tratase (art. 50.2). Si el empresario así lo decidiese, podría optar por la readmisión del trabajador, teniendo esté último derecho en dicho proceso a los salarios de tramitación correspondientes (art. 56.1 y 2).

En cuanto a la representación colectiva, en el art. 85.1 se otorga a los sindicatos un papel importante de negociación en el ámbito económico, laboral $\mathrm{y}$ sindical, y en cualquier otra materia que afecte a sus condiciones y/o relaciones de trabajo, entendiéndose que los representantes legales tienen la capacidad de mejorar las disposiciones mínimas establecidas tanto en la LPRL como en el ET. De igual manera, se contempla el derecho de los trabajadores de participar por medio de sus representantes legales en el centro de trabajo en la inspección y control de las medidas llevas a cabo por el empresario, siempre que no se cuenta con órganos o centros especializados en la materia.

\subsubsection{Ley General de la Seguridad Social}

En el caso que nos ocupa la declaración del daño causado por factores de riesgo psicosocial como contingencia común o profesional constituye una de las problemáticas esenciales en la materia que estamos analizando. La proclamación de enfermedad profesional o accidente de trabajo dota al sujeto de derechos extraordinarios de los que no se goza en el caso de declararse la contingencia como común (accidente no laboral o enfermedad común), tales como bases reguladoras más elevadas y periodo de carencia innecesario a efectos de prestaciones, indemnizaciones por, recargo de prestaciones, etc.

En líneas generales el legislador entiende que el accidente de trabajo es «toda lesión corporal que el trabajador sufra con ocasión o por consecuencia del trabajo que ejecute por cuenta ajena» (art. 115.1), presumiendo que es así salvo prueba en contrario (art. 115.3). Según esta definición los daños psicosociales no podrían ser declarados como tal, al no presentarse ninguna lesión corporal visible directamente. 
El art. 116, por su parte, establece que la enfermedad profesional es la contraída por la ejecución del trabajo por cuenta ajena en las actividades especificadas por medio del Real Decreto 1299/2006, de 10 de noviembre, por el que se aprueba el nuevo cuadro de enfermedades profesionales. Mediante este $\mathrm{RD}$ se ha configurado una lista de actividad-causa-daño científicamente avalados y sin admisión de prueba en contra (presunción iuris et de iure). Podemos decir que esta lista está bastante enfocada al sector industrial, no incluyéndose en la misma ningún factor proveniente de procesos productivos, elementos organizacionales ni características palpables del trabajo. Ni siquiera se contemplan en el Anexo 2, en el que la lista tradicional supera sus limitaciones del enfoque tradicional de la prevención, y elabora una lista de enfermedades sospechosas de conformidad a la Recomendación 2003/670/CE. Parece que la razón de su exclusión recae en carácter multifactorial de este tipo de riesgos, como bien hemos podido ver en apartados anteriores.

En esta situación, debemos intentar encajar los daños derivados en alguna de las otras situaciones previstas por la ley, dado que la imposibilidad de consonancia con los supuestos anteriores nos llevaría a declararlos como enfermedad común (art. 117.2), o en su caso, como accidente no laboral (art. 117.1). Por lo general, la jurisprudencia vislumbra una tendencia a declarar los daños psicosociales como enfermedad de trabajo (pero no profesional) fundamentándose en el art. 115.2.e $)^{24}$. A pesar de este reconocimiento, el trabajador se ve más vulnerable en el proceso judicial al tener que aportar pruebas que demuestren que la patología proviene únicamente de su labor por cuenta ajena.

\section{Posibles lineas de actuación}

Partiendo de la premisa de que nos encontramos en una etapa de mundialización de la economía, y de su consecuente y necesario cambio de paradigma de las relaciones laborales, intentaremos proponer medidas tendentes a minimizar los riesgos a los que se exponen los trabajadores, y buscar nuevas formar de actuación que contribuían a crear una organización de trabajo segura y sostenible tanto para trabajadores como para empresarios.

\subsection{Cambio parcial del modelo regulador de los riesgos psicosociales}

En nuestro país contamos con un modelo regulador centralista, es decir, las decisiones vinculantes emanan del Estado Central, concediendo a las CCAA únicamente el poder de desarrollar propuestas y líneas de actuación favorecedoras pero no de obligado cumplimiento.

24 Véanse, entre otras: STSJ País Vasco de 2 de noviembre de 1999 AS 4212, STSJ País Vasco de 9 de mayo de 2000 AS 3289, STSJ País Vasco de 7 de septiembre de 2004 AS 2005. 
Por otro lado, pese a que la ley reconoce que la negociación colectiva tiene capacidad para superar las disposiciones mínimas establecidas por la ley, en la práctica, los convenios se limitan en su mayoría a hacer una breve reseña a la LPRL. Se debe proporcionar mayores mecanismos a los representantes legales de los trabajadores para favorecer el consenso con los empresarios para desarrollar un mayor compromiso con la protección de la salud mental y psíquica de los trabajadores.

En líneas generales, se propone cambiar parcialmente el mecanismo de protección de riesgos en España, descentralizando la capacidad de establecer preceptos, que siempre dentro de la coordinación y concordancia con la LPRL, se adecuen de manera más exacta y eficaz a la problemática de sectores específicos y de tejidos productivos predominantes en lugares geográficos concretos. Para esto, se debe fortalecer el poder de la negociación colectiva, hoy en día en decrecimiento, y de igual manera, posibilitar que las comunidades autónomas tengan capacidad de elaborar acuerdos marcos vinculantes de fomento para todos los agentes implicados.

Así, se conseguiría instaurar un modelo de promoción de la prevención de riesgos laborales basado tanto en políticas explicativas como en políticas de fomento y promoción definidas.

\subsection{Fomento de la economía social y solidaria}

Una de las alternativas más viables para superar los problemas laborales ocasionados por la flexibilidad enmarcada en la economía actual se centra en construir una sociedad que promueva la economía social y solidaria mediante la creación de cooperativas y sociedades laborales. Se trata de superar el concepto de empresa instaurado por la teoría clásica y desviar el tejido productivo a formas de producción ahora minoritarias, que sustituyan la asociación de capitales anónimos por la de personas visibles.

Este tipo de economía, principalmente difundida a través de cooperativas y sociedades laborales, se caracteriza principalmente por concentrar en el mismo grupo de personas la propiedad de la organización y la fuerza de trabajo, por una gestión autónoma y democrática, y por unos principios arraigados de solidaridad, justicia y cooperación.

Las organizaciones que funcionan en base a estos principios se sustentan en unos valores compartidos por todos sus miembros, los cuales participan de manera equitativa, activa y libre en la gestión y toma de decisiones (una persona-un voto). De esta manera, los empresarios-trabajadores se involucran y se comprometen con la realidad productiva y organizativa, y se generan mecanismos naturales de transmisión de información y conocimiento dentro de un marco notable de seguridad y estabilidad laboral. Al mismo tiempo, las diferencias salariales de sus integrantes tiendes a ser más justas y menos diferenciales. 
Asimismo, el compromiso contraído con la totalidad de la sociedad (más allá de la consecución de beneficios económicos) no solo contribuye a solucionar problemas sociolaborales a nivel organizacional, sino que sortea la generación de una posible imagen externa negativa derivada de una gestión poco ética.

Como podemos apreciar, la economía social y solidaria proporciona una solución a mucho de los problemas ocasionados por la precariedad laboral. La falta de participación, control y comunicación; los conflictos de rol y el desconocimiento del responsable de las obligaciones; y la inseguridad y la falta de expectativas de desarrollo profesional serían suprimidas en aras a constituir una organización y condiciones de trabajo pactadas por los trabajadores y para los trabajadores.

En este sentido, y pese a que ya se han llevado a cabo medidas de fomento de cooperativas y sociedades laborales, las administraciones públicas deben plantear iniciativas más eficaces y concretas para favorecer la creación de organizaciones de carácter social y solidario. Se proponen las siguientes actuaciones: Configurar un conjunto de facilidades de financiación para los emprendimientos de esta naturaleza y sus procesos de evaluación y acompañamiento necesarios, posibilitar créditos personales orientados al consumo de bienes y servicios de entidades de la economía social y solidaria, fomentar una cultura del emprendimiento y del autoempleo social y solidario, propagar los valores afines desde edades tempranas e incluir como criterio positivo la naturaleza social y solidaria de las empresas y entidades que participen en procesos de contrataciones públicas, ya sea para concursos como para concesión de subvenciones.

\subsection{Mayor presencia de los riesgos psicosociales en la legislación}

Tomando como punto de referencia el análisis jurídico realizado en el apartado precedente, y centrándonos en la legislación existente en nuestro país, se aprecia una necesidad de intensificación de la presencia de los riesgos psicosociales como elemento susceptible de ser prevenido y reparado:

- Inclusión de una referencia completa a los riesgos psicosociales en la LRPL para fomentar que los encargados de garantizar la protección fueran más conscientes de la existencia de estos riesgos, y que los equiparasen (no solo en la ley) al resto de los factores «tradicionales» a nivel práctico.

- Creación nuevas figuras e instrumentos organizacionales que complementases a la figura del experto en psicosociología en los servicios de prevención de las empresas., como por ejemplo consejeros psicosociales y sistemas de gestión del estrés.

- Aumento de la periodicidad de las auditorías de 4 a 2 años, y anualmente en los casos en los que la actividad sea considerada de riesgo especial. 
- En el plano reparador, creación de una lista de similar magnitud a la establecida por el Real Decreto 1299/2006, incluyendo una serie de riesgos psicosociales asociados a ciertos sectores concretos que cuentan con una fiabilidad científica desarrollada y susceptible de ser modificada para agilizar su reconocimiento en los juzgados, evitando así largos procesos judiciales.

\subsection{Incentivos a la Responsabilidad Social Empresarial (RSE)}

El Foro de Expertos en RSE del Ministerio de Trabajo define la responsabilidad social empresarial como, la integración voluntaria por parte de la empresa, en su gobierno y gestión, en su estrategia, políticas y procedimientos, de las preocupaciones sociales, laborales, medioambientales y de respeto a los derechos humanos que surgen de la relación y el diálogo transparentes con sus grupos de interés, responsabilizándose así de las consecuencias y de los impactos que se derivan de sus acciones, más allá del cumplimiento estricto de las obligaciones legales vigentes ${ }^{25}$.

De esta definición se concluye que la RSE se trata de un compromiso adquirido por las empresas con el entorno, tanto externo como interno, para la mejora de la sociedad. Sin embargo, el compromiso adquirido por las empresas socialmente responsables generan una imagen exterior que puede ser identificada como valor añadido para la empresa.

La RSE, en su dimensión interna, tiene en cuenta las buenas prácticas en la gestión de las personas dentro de la organización. Podríamos decir que una empresa socialmente responsable dispone de una política de recursos humanos de calidad, englobando en ella adecuadas líneas de actuación en procesos de contratación y formación, seguridad y salud laboral e igualdad y conciliación.

La Ley 2/2011, de 4 de marzo, de Economía Sostenible, desarrollada para la instauración de una economía respetuosa con el medio ambiente y favorecedora del empleo de calidad, la igualdad de oportunidades y la cohesión social, recoge la intención de los organismos públicos de favorecer la colaboración con entidades de responsabilidad social y de reconocer la labor de las mismas (arts. 35 y 39).

Esta Ley, que supone un avance considerable para el reconocimiento de la RSE en nuestra sociedad, debe ser complementada por acciones que tiendan a crear una cultura de normalización de la responsabilidad de las empresas con la sociedad. Ejemplo de esas acciones podrían ser la elaboración de guías de Buenas Prácticas de RSE para cada sector económico y para Comunidad Autónoma,

25 Foro de expertos sobre RSE (2007). Informe del foro de expertos en responsabilidad social de las empresas, Madrid, Ministerio de Empleo y Seguridad Social. 
fomento de la divulgación de los valores y la cultura de la RSE, impulso de acciones informativas que permitan fortalecer la cultura de un consumo responsable de los ciudadanos como consumidores, y la Incorporación de la RSE en el diseño de los instrumentos y herramientas de apoyo a emprendedores.

\subsection{Equidad salarial y buenas prácticas en las Administraciones Públicas}

Por último, debemos hacer una mención especial al papel esencial que debieran realizar las Administraciones Públicas en la consecución de un sistema de relaciones laborales digno y justo.

En este sentido, la realidad nos muestra que la actuación de las Administraciones Públicas no es meritoria. Por un lado, las diferencias salariales en los organismos públicos son incluso mayores a los de las empresas privadas, especialmente discriminatorias en mujeres, niveles jerárquicos bajos y nuevas incorporaciones; Por otro lado, los procesos de licitación regulados en el Decreto Legislativo 3/2011, de 14 de noviembre, por el que se aprueba el texto refundido de la Ley de Contratos del Sector Público permiten que los contratistas puedan subcontratar hasta un $60 \%$ del importe de la adjudicación, incluso dejando libertad a que esa cantidad pueda ser superada en los pliegos.

Los organismos públicos deben mostrar una actuación ejemplar a los ciudadanos y contribuir a la generación de un empleo estable y de calidad modificando la regulación de la estructura salarial (tanto del funcionariado como del personal laboral) y la de los contratos públicos.

\section{Bibliografía}

Álvarez Cuesta, Henar (2008). La precariedad laboral: análisis y propuestas de solución, Albacete, Bomarzo.

Boada-Grau, Joan y Ficapal-Cusí, Pilar (2012). Salud y trabajo. Los nuevos riesgos, Barcelona, UOC.

Brun, Emmanuelle y MilczareK, Malgorzata (2007). Expert forecast on emerging psychosocial risks related to occupational safety and health, Bélgica, Agencia Europea de Salud y Seguridad en el Trabajo.

Comisión de Trabajo y Asuntos Sociales (2006). Informe de la subcomisión para potenciar y promover la responsabilidad social de las empresas, Madrid, Palacio del Congreso de los Diputados.

Comisión Europea (2014). Communication from the Commission to the European Parliament, the council, the European Economic and Social Committee and the Committee of the Regions on an eu strategic framework on health and safety at work 2014-2020, Bruselas, Parlamento Europeo.

Comité Europeo de Derechos Sociales (2008). «All workers have the right to a fair remuneration sufficient for a decent standard of living for themselves and their families», Digest of the case law of the european committee of social rights, 4, 43-49. 
Del Hoyo Delgado, Maria Ángeles (2004). Estrés laboral, Madrid, INSHT.

Díez de Castro, José; Redondo López, Carmen y Rivas Costa, Javier (2001). «Flexibilidad numérica en las empresas: el trabajo a tiempo parcial en Galicia», Revista Galega de Economía de la Universidad de Santiago de Compostela, vol. 10, 2.

Evans, John y Gibb, Euan (2009). "El Cambio del Empleo Precario al Trabajo Decente», en Global Union Research Network, Ginebra, OIT.

FORO DE EXPERTOS SOBRE RSE (2007). Informe del foro de expertos en responsabilidad social de las empresas, Madrid, Ministerio de Empleo y Seguridad Social.

Gobierno Vasco (2015). Estrategia Vasca de Seguridad y Salud en el Trabajo para el periodo 2015-2020, Vitoria, Departamento de Empleo y Políticas Sociales. http://www. elmundo.es/elmundosalud/2012/09/19/neurociencia/1348056989.html

Instituto InTERnacional de Estudios Laborales (2009). Informe sobre el trabajo en el mundo 2009: Crisis mundial del empleo y perspectivas, Ginebra, OIT.

Jaramillo Osorio J. y Aponte, José Ignacio (2013). Papel de gestión humana frente a la flexibilidad salarial en organizaciones colombianas, Colombia, Universidad de Manizales Facultades de Ciencias Sociales y Humanas.

Laparra Navarro, Miguel (2006). La construcción del empleo precario: dimensiones, causas $y$ tendencias de la precariedad laboral, Madrid, Cáritas Española.

McGrath, J.E. (1907): "A conceptual formulation for research on stress», en Social and psychological factors in stress, Nueva York, 10-21.

OIT (1984): «Los factores psicosociales en el trabajo: naturaleza, incidencia y prevención», Informe del Comité Mixto OIT-OMS sobre Medicina del Trabajo, novena reunión, Ginebra.

Ortiz García, Pilar (2013): «Cambios en la legislación laboral y contratación temporal en España», Cuadernos de Relaciones Laborales, Vol. 31, 1, 141-165.

Red De Economía Alternativa y Solidaria (2014). Resumen del I congreso internacional de economía social y solidaria: la economía se encuentra con las personas, Zaragoza.

Somavía, Juan (1999). Trabajo Decente: Memoria del Director General. Ginebra, OIT.

UE (2008). Pacto Europeo Para la Salud Mental y el Bienestar. Bruselas, Conferencia de alto nivel de la UE. 